\title{
The impacts of thyroid function on the diagnostic accuracy of Cystatin $C$ to detect acute kidney injury in ICU patients: a prospective, observational study
}

\author{
Feilong Wang ${ }^{1+}$, Wenzhi Pan ${ }^{2 \dagger}$, Hairong Wang ${ }^{1+}$, Yu Zhou ${ }^{1}$, Shuyun Wang ${ }^{1}$ and Shuming Pan ${ }^{1 *}$
}

\begin{abstract}
Introduction: Cystatin C (Cysc) could be affected by thyroid function both in vivo and in vitro and thereby may have limited ability to reflect renal function. We aimed to assess the association between Cysc and thyroid hormones as well as the effect of thyroid function on the diagnostic accuracy of Cysc to detect acute kidney injury (AKI).

Methods: A total of 446 consecutive intensive care unit (ICU) patients were screened for eligibility in this prospective AKI observational study. Serum Cysc, thyroid hormones and serum creatinine (Scr) were measured upon entry to the ICU. We also collected each patient's baseline characteristics including the Acute Physiology and Chronic Health Evaluation II (APACHE-II) score. The diagnostic performance of Cysc was assessed from the area under the receiver operator characteristic curve (AUC) in each quartile of thyroid hormone(s).

Results: A total of 114 (25.6\%) patients had a clinical diagnosis of AKI upon entry to the ICU. The range of free thyroxine (FT4) value was 4.77 to $39.57 \mathrm{pmol} / \mathrm{L}$. Multivariate linear regression showed that age (standardized beta $=0.128, P<0.0001$ ), baseline Scr level (standardized beta $=0.290, P<0.0001$ ), current Scr (standardized beta $=0.453, P<0.0001$ ), albumin (standardized beta $=-0.086, P=0.006$ ), and FT4 (standardized beta $=0.062$, $P=0.039$ ) were related with Cysc. Patients were divided into four quartiles based on FT4 levels. The AUC for Cysc in detecting AKI in each quartile were as follows: 0.712 in quartile I, 0.754 in quartile II, 0.829 in quartile III and 0.797 in quartile IV. There was no significant difference in the AUC between any two groups (all $P>0.05$ ). The optimal cut-off value of Cysc for diagnosing AKI increased across FT4 quartiles $(1.15 \mathrm{mg} / \mathrm{L}$ in quartile $\mathrm{I}, 1.15 \mathrm{mg} / \mathrm{L}$ in quartile II, $1.35 \mathrm{mg} / \mathrm{L}$ in quartile III and $1.45 \mathrm{mg} / \mathrm{L}$ in quartile IV).
\end{abstract}

Conclusions: There was no significant impact of thyroid function on the diagnostic accuracy of Cysc to detect AKI in ICU patients. However, the optimal cut-off value of Cysc to detect AKI could be affected by thyroid function.

\section{Introduction}

Acute kidney injury (AKI) is a prevalent problem and still a big challenge to both the developed and developing world [1]. About two-thirds of intensive care unit (ICU) patients develop an episode of AKI during their ICU stay [2]. Both short-term and long-term mortality were higher in ICU patients with AKI than those without [3-5]. Studies have found that early detection and treatment of AKI may improve outcomes [2]. Thus,

\footnotetext{
* Correspondence: pansm2010@sina.cn

${ }^{\dagger}$ Equal contributors

'Department of Emergency, Xinhua Hospital Affiliated to Shanghai Jiaotong University School of Medcine, NO.1665, Kongjiang Road, Shanghai 200092, China

Full list of author information is available at the end of the article
}

timely diagnosis of AKI development after renal insult is urgent. Cystatin C (Cysc), a 13-kDa cysteine proteinase inhibitor, is freely filtered at the glomerulus and neither secreted nor reabsorbed by renal tubules. This physiological feature makes Cysc an ideal glomerular filtration biomarker. During the past few years, some studies have focused on the potential value of Cysc for the diagnosis and early detection of AKI [6-14]. However, these studies have reported conflicting results. Some studies reported good discrimination for Cysc in the early detection of AKI in various patient populations [7-9,14,15], while other studies found that Cysc had poor or moderate ability to predict AKI [6,10-13]. Besides that, there was no consensus about the appropriate cut-off value for using Cysc to diagnose or predict AKI [6-14,16]. These inconsistent

\section{Biomed Central}


results limit the usefulness of Cysc in the early detection of AKI in clinical practice.

Cysc is produced by all nucleated cells in the human body at a relatively constant rate [17]. However, recent studies found that thyroid hormones could stimulate the production of Cysc in vitro $[18,19]$. Moreover, clinical studies also found that Cysc was significantly associated with thyroid function [20-24]. The impact of thyroid hormones on the diagnostic value of Cysc in detecting AKI has raised concerns in clinical practice [25-27]. To the best of our knowledge, whether thyroid hormones are related to the level of Cysc in ICU patients has never been investigated. The effect of thyroid hormones on the diagnostic accuracy and threshold of Cysc in predicting AKI has also not been defined.

Therefore, we undertook a prospective, observational study in a large population of unselected ICU patients to assess: 1) the relationship between Cysc and thyroid hormones; and 2) the effect of thyroid function on the diagnostic value of Cysc in detecting AKI.

\section{Material and methods Participants}

This prospective study recruited consecutive patients 18 years old and older hospitalized in the ICU of Xinhua Hospital affiliated with Shanghai Jiaotong University School of Medicine between April 2011 and May 2012, including medical and trauma patients. We decided $a$ priori to exclude patients according to the following criteria: 1) past history of any thyroid diseases, such as hyperthyroidism, hypothyroidism and thyroid tumors; 2) thyroid nodule found by physical examination when admitted to ICU; 3) pregnancy within the previous six months; 4) undergoing any hormone replacement therapy except insulin use; 5) pre-existing severe renal disease (serum creatinine $(\mathrm{Scr})>300 \mathrm{umol} / \mathrm{L}$ ) or pre-existing dialysis; and 6) undergoing continuous renal replacement therapy (CRRT) in the four weeks before the blood sample was collected. Patients who died or were discharged from the ICU within four hours of admission were also excluded because data collection was difficult for these patients. The Shanghai Jiaotong University Xinhua Hospital Ethics Committee approved the study and waived the requirement for informed consent, because this was an observational study and all laboratory indices observed were commonly measured for all patients in our ICU department.

\section{Definition of acute kidney injury}

The patients were diagnosed as having AKI by using the stage 1 AKI criteria of the Acute Kidney Injury Network (AKIN) classification: new-onset of at least 1.5-fold increase or $\geq 0.3 \mathrm{mg} / \mathrm{dL}(26.5 \mathrm{umol} / \mathrm{L})$ increment of $\mathrm{SCr}$ from baseline. If the preadmission Scr was available, we defined baseline creatinine by using the following rules ranked in descending order of preference: 1) the lowest value between three and 365 days preadmission; 2) the lower value of either preadmission Scr within three days preadmission or the lowest Scr measurement in seven days after admission to the ICU, if it was less than the initial creatinine on entry to the ICU; and 3) the lowest value of preadmission Scr within three days. If the value of 2) and 3) were greater than the initial creatinine on entry to the ICU or if the preadmission value was unavailable, then the lower value of either the initial creatinine on entry to the ICU or the lowest $\mathrm{pCr}$ measurement in seven days was used. We did not use the Modification of Diet in Renal Disease (MDRD) formula to back-calculate baseline creatinine because using this method to estimate baseline creatinine would result in an overestimation of the prevalence of AKI [28].

\section{Clinical data and laboratory methods}

We prospectively collected each patient's demographic and clinical characteristics, including the Acute Physiolgy and Chronic Health Evaluation II (APACHE-II) score (which can range from 0 to 71, with higher scores indicating more severe illness). Serum Cysc, creatinine, glucose, lactate, electrolytes and albumin levels were measured using a Hitachi 7600-120 analyzer (Hitachi, Tokyo, Japan). The analytical range of Cysc in the laboratory of our hospital is 0.4 to $7.5 \mathrm{mg} / \mathrm{L}$. The reported total coefficients of variation are $4.2 \%$ at mean concentrations $0.6 \mathrm{mg} / \mathrm{L}$ and $3.8 \%$ at $1.4 \mathrm{mg} / \mathrm{L}$, respectively. Thyroid hormones, including free triiodothyronine (FT3), free thyroxine (FT4), total triiodothyronine (TT3), total thyroxine (TT4) and thyroid-stimulating hormone (TSH), were measured using the ADVIA Centaur immunoassay system (Siemens Healthcare Diagnostics Inc, Tarrytown, NY, USA). Reverse triiodothyronine (rT3) level was measured using the Maglumi 1000 Analyzer chemiluminescence immunoassay system (SNIBE Co, Ltd, Guandong, China). Serum C-reactive protein (CRP) levels were measured using the QuikRead CRP test kit (Orion Diagnostica, Espoo, Finland). We calculated the estimated glomerular filtration rate (eGFR) by using the abbreviated MDRD study equation. Blood samples were collected from all eligible patients when they were admitted to the ICU.

\section{Statistical analysis}

Continuous variable and categorical variables were presented as mean value $\pm \mathrm{SD}$ and precent, respectively. Bivariate correlation analysis was utilized to examine the association between two variables. Multivariate linear regression was performed to search for factors independently associated with Cysc. A criterion of $P<0.05$ for entry and $P \geq 0.10$ for removal was imposed in this procedure. We divided FT4 into quartiles and compared 
demographics, clinical characteristics and laboratory test results with analysis of variance for continuous variables and chi-square or Fisher's exact tests for categorical variables. Receiver operator characteristic (ROC) curve was used to examine the performance of Cysc to detect AKI. The curve represented a plot of sensitivity versus 1specificity. The area under the curve (AUC) was derived from the ROC curve. The differences between AUC were tested by Hanley - McNeil methods [29]. A statistically derived value, based on the Youden index16, maximizing the sum of the sensitivity and specificity was used to define the optimal cut-off value. A two-sided $P$ value $<0.05$ was considered to indicate statistical significance. All analyses were performed with SPSS 13.0 software.

\section{Results}

\section{Base-line characteristics and base-line factors related to} Cysc

Cysc and thyroid hormones were missed in 12 patients. Those patients were excluded from the study. A total of 446 patients were screened for eligibility in this analysis. The mean age was $69.07 \pm 15.58$ years and $58.3 \%$ were men. Mean Apache-II score was $15.46 \pm 7.34$. On entry to the ICU, 114 (25.6\%) had a clinical diagnosis of AKI. A total of 56 patients died during their ICU stay. In Table 1, bivariate correlation analysis shows that a higher level of Cysc was associated with older age, higher level of baseline Scr and current Scr, higher Apache-II score and lower level of albumin. In addition, there was a negative association between Cysc and TT3 as well as FT3, and a positive association between Cysc and FT4. In

Table 1 Factors associated with Cysc by bivariate correlation analysis

\begin{tabular}{lcc}
\hline Variables & \multicolumn{2}{c}{ Cysc } \\
\cline { 2 - 3 } & $\mathbf{R}$ & $\boldsymbol{P}$ \\
\hline Current Scr (umol/L) & 0.684 & 0.000 \\
Baseline Scr (umol/L) & 0.562 & 0.000 \\
Sex & 0.027 & 0.568 \\
Age (years) & 0.380 & 0.000 \\
Albumin (g/L) & -0.230 & 0.000 \\
TT3 (nmol/L) & -0.099 & 0.036 \\
TT4 (nmol/L) & 0.005 & 0.916 \\
FT3 (pmol/L) & -0.180 & 0.000 \\
FT4 (pmol/L) & 0.098 & 0.039 \\
TSH (IU/mL) & 0.168 & 0.054 \\
rT3 (nmol/L) & -0.005 & 0.911 \\
APACHE-II score & 0.429 & 0.000 \\
\hline APACHE I/score, Acte Physolog and Chro &
\end{tabular}

APACHE II score, Acute Physiology and Chronic Health Evaluation II score; Baseline Scr, baseline serum creatinine; Current Scr, serum creatinine on the entry to ICU; FT3, free triiodothyronine; FT4, free thyroxine; $\mathrm{rT3}$, reverse triiodothyronine; $\mathrm{TSH}$, thyroid-stimulating-hormone; $\Pi 3$, total triiodothyronine; $\Pi 4$, total thyroxine. multivariate linear regression (Table 2), age (standardized beta $=0.128, P<0.0001$ ), baseline Scr level (standardized beta $=0.290, P<0.0001$ ), current Scr (standardized beta $=$ $0.453, P<0.0001$ ), albumin (standardized beta $=-0.086$, $P=0.006$ ), and FT4 (standardized beta $=0.062, P=0.039$ ) were related to Cysc.

Differences in clinical and laboratory characteristics among the quartiles of FT4 are listed in Table 3. Patients in the higher quartiles of FT4 tended to be older and had higher levels of albumin, TT4 and FT3, and a low level of TSH. However, there was no significant difference among the four quartiles in other clinical and laboratory characteristics, including sex, level of baseline Scr, current Scr, Cysc, TT3, rT3, APACHE-II score as well as the prevalence of AKI.

\section{Association between thyroid hormones and APACHE-II scores}

Among the thyroid hormones, only FT4 was not related to APACHE-II scores $(P=0.219)$. FT3 $(\mathrm{r}=-0.363, P<0.001)$, TT3 $(\mathrm{r}=-0.138, P<0.001)$, TT4 $(\mathrm{r}=-0.338, P<0.001)$, TSH $(\mathrm{r}=-0.133, P=0.005)$ and $\mathrm{rT} 3(\mathrm{r}=-0.205, P<0.001)$ were all correlated with APACHE-II scores.

\section{Association between Cysc and AKI in patients with different levels of FT4}

To evaluate the impact of FT4 on the accuracy of Cysc in the diagnosis of AKI, a ROC curve was drawn in each quartile of FT4 (Figure 1). The AUC was calculated as 0.712 in quartile I, 0.754 in quartile II, 0.829 in quartile III and 0.797 in quartile IV (Table 4). There was no significant difference in AUC between any two groups. The optimal cut-off value of Cysc for diagnosing AKI increased across FT4 quartiles (quartile I and quartile II $=1.15 \mathrm{mg} / \mathrm{L}$, quartile III $=1.35 \mathrm{mg} / \mathrm{L}$, quartile IV $=1.45 \mathrm{mg} / \mathrm{L}$ ).

Table 2 Factors associated with Cysc in multivariate linear regression

\begin{tabular}{lcc}
\hline $\begin{array}{l}\text { Independent } \\
\text { variables }^{\mathrm{a}}\end{array}$ & \multicolumn{2}{c}{ Cysc } \\
\cline { 2 - 3 } & Standardized $\boldsymbol{\beta}$ & $\boldsymbol{P}$ \\
\hline Current Scr (umol/L) & 0.486 & 0.000 \\
Baseline Scr (umol/L) & 0.290 & 0.000 \\
Age (years) & 0.128 & 0.000 \\
Albumin (g/L) & -0.086 & 0.006 \\
FT4 (pmol/L) & 0.062 & 0.039 \\
Constant & 0.291 (Unstandardized) & 0.109
\end{tabular}

andependent variables included age, sex, baseline Scr, current Scr, albumin, $\Pi \pi 3, T T 4$, FT3, FT4, TSH. Variables not listed in the table were removed from the stepwise analysis. Adjusted R square 0.627. Baseline Scr, baseline serum creatinine; Current Scr, serum creatinine on the entry to ICU; FT3, free triiodothyronine; FT4, free thyroxine; rT3, reverse triiodothyronine; TSH, thyroid-stimulating-hormone; $\Pi 3$, total triiodothyronine; $\Pi 4$, total thyroxine. 
Table 3 Baseline clinical and laboratory characteristics by quartile of FT4

\begin{tabular}{|c|c|c|c|c|c|}
\hline & $\mathrm{I}$ & II & III & IV & $P$ \\
\hline Number & 111 & 111 & 112 & 112 & / \\
\hline Age (years) & $65.2 \pm 16.7$ & $69.4 \pm 15.6$ & $71.3 \pm 15.1$ & $70.3 \pm 14.4$ & 0.020 \\
\hline Men, Number (\%) & 65 (58.6) & $67(60.4)$ & $72(64.3)$ & $56(50.0)$ & 0.171 \\
\hline APACHE-II score (points) & $15.6 \pm 7.3$ & $15.1 \pm 7.9$ & $14.9 \pm 6.7$ & $16.3 \pm 7.4$ & 0.524 \\
\hline Baseline Scr (umol/L) & $80.0 \pm 45.9$ & $80.0 \pm 39.0$ & $86.6 \pm 46.4$ & $84.0 \pm 48.7$ & 0.636 \\
\hline Current Scr (umol/L) & $98.2 \pm 89.4$ & $100.7 \pm 61.5$ & $108.1 \pm 79.7$ & $106.8 \pm 76.1$ & 0.741 \\
\hline Cysc (mg/L) & $1.23 \pm 0.65$ & $1.23 \pm 0.51$ & $1.32 \pm 0.67$ & $1.35 \pm 0.79$ & 0.354 \\
\hline Albumin (g/L) & $32.7 \pm 4.7$ & $35.0 \pm 6.1$ & $35.1 \pm 5.0$ & $35.4 \pm 5.5$ & 0.000 \\
\hline TT3 (nmol/L) & $0.83 \pm 0.39$ & $0.94 \pm 0.34$ & $0.89 \pm 0.29$ & $0.90 \pm 0.36$ & 0.109 \\
\hline TT4 (nmol/L) & $72.04 \pm 28.93$ & $89.17 \pm 23.00$ & $90.03 \pm 20.98$ & $94.32 \pm 24.29$ & 0.000 \\
\hline FT3 (pmol/L) & $3.04 \pm 0.62$ & $3.27 \pm 0.54$ & $3.42 \pm 0.59$ & $3.66 \pm 0.92$ & 0.000 \\
\hline FT4 (pmol/L) & $11.93 \pm 1.54$ & $14.47 \pm 0.53$ & $16.14 \pm 0.50$ & $19.58 \pm 3.17$ & 0.000 \\
\hline TSH (IU/mL) & $3.87 \pm 16.16$ & $1.26 \pm 1.03$ & $1.70 \pm 1.84$ & $1.14 \pm 1.05$ & 0.044 \\
\hline rT3 (nmol/L) & $0.49 \pm 0.21$ & $0.54 \pm 0.21$ & $0.54 \pm 0.24$ & $0.57 \pm 0.27$ & 0.113 \\
\hline AKI (Number, \%) & $24(21.6)$ & $26(23.4)$ & $36(32.1)$ & $28(25.0)$ & 0.292 \\
\hline
\end{tabular}

Quartile cut points for FT4 were 13.49, 15.38 and 17.07 pmol/L, continuous variables were compared with one-way ANOVA, and dichotomous variables were compared with the chi-square test. ANOVA, analysis of variance; APACHE II score, Acute Physiology and Chronic Health Evaluation II score; Baseline Scr, baseline serum creatinine; Current Scr, serum creatinine on the entry to ICU; Cysc, Cystatin C; FT3, free triiodothyronine; FT4, free thyroxine; rT3, reverse triiodothyronine; $\mathrm{TSH}$, thyroid-stimulating-hormone; $\mathrm{T} 3$, total triiodothyronine; TT4, total thyroxine.

\section{Discussion}

In this prospective study, we first assessed the impact of thyroid function on the accuracy of Cysc in the diagnosis of AKI in ICU patients. The primary findings of this study are as follows: (1) FT4 was the only index among thyroid hormones that was not correlated with APACHEII score and still related to the Cysc level after adjusting for several confounding factors; (2) there was no significant difference in the diagnostic performance of Cysc among patients with different FT4 levels; and (3) the optimal cut-off value of Cysc in diagnosing AKI increased as FT4 increased.

Previous studies have shown that some thyroid hormones might be confounded by critical illness and a low FT3 level was correlated with the severity of morbidity and the outcomes of patients in ICUs [30,31]. Our present study also confirmed the negative association between FT3 level and APACHE-II score. As we know, the severity of illness score was positively associated with the risk of AKI as well as the level of Cysc, and thus FT3 was inversely related to Cysc. However, the level of FT4 was the only index among thyroid hormones that was not related to the APACHE-II score in this study. This indicated that FT4 was less affected by the severity of illness and could reflect a stable state of thyroid hormones as compared with other indices of thyroid hormones. So, FT4 can directly reflect the stimulating effect of thyroid hormones on Cysc and FT4 was positively related to Cysc. In fact, FT4 was the only index independently correlated with Cysc. Based on these results, we chose FT4 as the representative variable to assess the impact of thyroid function on the accuracy of Cysc in the diagnosis of AKI in ICU patients.

Cysc used to be considered less subject to the nonrenal variables that impact Scr. However, more recent studies [18-24,32] have found that several factors are associated with higher Cysc in noncritically ill patients, including older age, male sex, current smoking status, elevated CRP levels as well as thyroid hormones. However, the factors associated with the Cysc level were not well defined in critically ill patients. In a study of 85 ICU patients, the investigator reported that the Cysc level was not affected by low $\mathrm{T} 3$ or $\mathrm{T} 3 / \mathrm{T} 4$ syndrome [6]. In contrast with this study, we found that Cysc level was not only associated with both baseline Scr and current Scr levels, but also is related to age, and levels of albumin and FT4 in ICU patients.

Results relating to the diagnostic accuracy of Cysc in detecting AKI have been conflicting in previous studies. The AUC for Cysc ranged from 0.55 to 0.99 across different studies [16]. Some previous studies have found that thyroid hormones were associated with Cysc level both in vitro [18,19] and in vivo [20-24], but whether the thyroid function could affect the performance of Cysc to identify AKI was never investigated before. Our present study showed that the difference of AUC among different FT4 quartiles did not reach statistical significance. Although thyroid hormones could stimulate the production of Cysc at baseline as well as the progress of AKI after a critical illness, the acute decrease of GFR during 
A

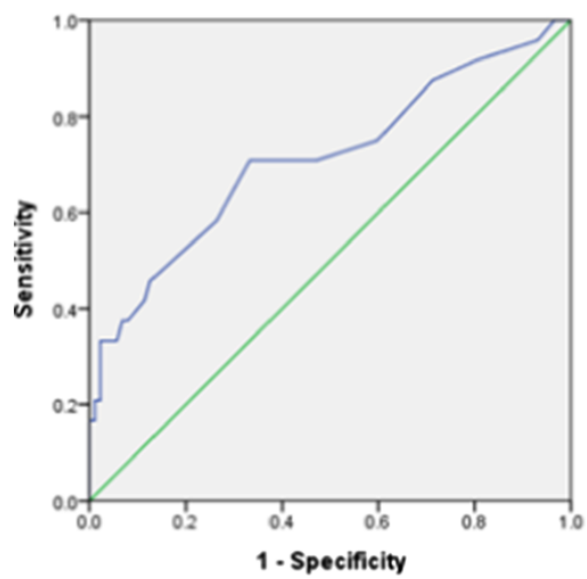

C

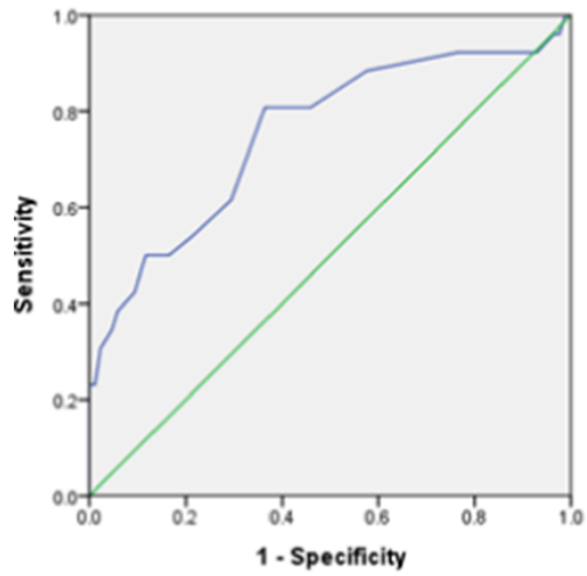

B

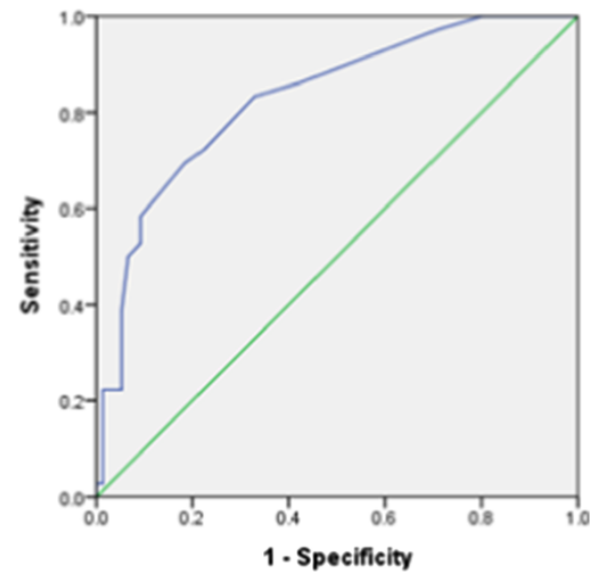

D

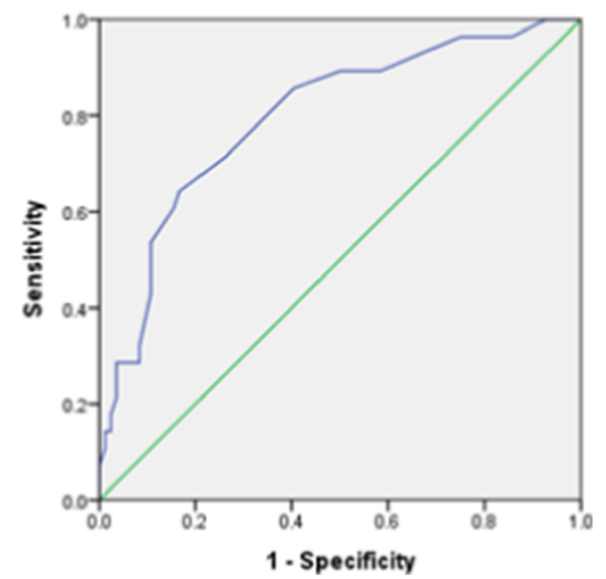

Figure 1 Receiver operating characteristic curves for Cysc in quartile I (A), quartile II (B), quartile III (C) and quartile IV (D).

critical illness was the most important factor for the increment of Cysc from baseline. This may be the reason. However, the optimal cut-off value of Cysc in the diagnosis of AKI increased with increasing FT4 quartile is an interesting finding. This result indicates that FT4 might stimulate Cysc production and, thus, affect the absolute value of Cysc and the optimal cut-off value of

Table 4 Performance of Cysc in diagnosing AKI by quartile of FT4

\begin{tabular}{cccccc}
\hline & AUC ROC & $\mathbf{9 5 \% ~ C l}$ & Cut-off & Sensitivity & Specificity \\
\hline I & $0.712 \pm 0.066$ & $0.582-0.841$ & 1.15 & 0.708 & 0.667 \\
II & $0.754 \pm 0.059$ & $0.638-0.870$ & 1.15 & 0.808 & 0.635 \\
III & $0.829 \pm 0.041$ & $0.748-0.910$ & 1.35 & 0.694 & 0.816 \\
IV & $0.797 \pm 0.049$ & $0.700-0.893$ & 1.45 & 0.643 & 0.833 \\
\hline
\end{tabular}

Quartile I versus Quartile II $Z=0.474, P=0.318$; Quartile I versus Quartile III $Z=1.506, P=0.066$; Quartile I versus Quartile IV $Z=1.034, P=0.151$; Quartile II versus Quartile III $Z=1.044, P=0.148$; Quartile II versus Quartile IV $Z=0.561$, $P=0.288$ ]. Quartile III versus Quartile IV $Z=0.501, P=0.308$. AUC ROC, area under the receiver operating characteristic curve; $\mathrm{Cl}$, confidence interval.
Cysc in the diagnosis of AKI. This result may be one of the explanations fpr a wide range of optimal cut-off values of Cysc in detecting AKI in previous studies [16]. It also should be taken into consideration when using Cysc to identify AKI in clinical settings.

Several limitations to our study exist. First, we used Cysc to diagnose rather than predict AKI. We could not exclude the possibility that some non-AKI patients progressed to AKI after their entry into the ICU. However, whether Cysc could serve as an early biomarker of AKI remains uncertain. Second, because some unknown nonGFR determinants of Cysc may exist, the relationship between Cysc and thyroid hormones would be changed if adjusting for those confounding factors.

\section{Conclusions}

In this large-scale study of unselected ICU patients, we found that FT4 was the only index among thyroid hormones that was not correlated with APACHE-II score and still related to Cysc level after adjusting for several 
confounding factors. Thyroid function had no significant impact on the diagnostic accuracy of Cysc to detect AKI in ICU patients. However, the optimal cut-off value of Cysc to detect AKI could be affected by thyroid function.

\section{Key messages}

- FT4 was the only index among thyroid hormones that was not correlated with APACHE-II score and still related to Cysc level after adjusting for several confounding factors.

- There was no significant impact of thyroid function on the diagnostic accuracy of Cysc to detect AKI in ICU patients.

- The optimal cut-off value of Cysc to detect AKI could be affected by thyroid function.

\section{Abbreviations \\ AKI: acute kidney injury; APACHE-II score: Acute Physiology and Chronic Health Evaluation II score; AUC: area under the curve; Cysc: Cystatin C; eGFR: estimated glomerular filtration rate; FT3: free triiodothyronine; FT4: free thyroxine; GFR: glomerular filtration rate; NTIS: nonthyroidal illness syndrome; ROC: receiver operator characteristic; rT3: reverse triiodothyronine; TSH: thyroid-stimulating hormone; TT3: total triiodothyronine; TT4: total thyroxine.}

\section{Competing interests}

The authors declare that they have no competing interests.

\section{Authors' contributions}

FW and SP contributed to the design, data collection and manuscript writing. WP and YZ contributed to the design and statistical analysis. HW and SW participated in data collection. All authors read and approved the final manuscript.

\section{Acknowledgements}

This study was supported by Shanghai public health outstanding subject leader issue (GWDTR201219) and Shanghai Health Bureau (20124261). No conflict of interest exists. No relationship with industry exists.

\section{Author details}

'Department of Emergency, Xinhua Hospital Affiliated to Shanghai Jiaotong University School of Medcine, NO.1665, Kongjiang Road, Shanghai 200092, China. ${ }^{2}$ Department of Cardiology, Shanghai Institute of Cardiovascular Diseases, Zhongshan Hospital of Fudan University, NO.180, Fenglin Road, Shanghai 200032, China.

Received: 29 July 2013 Accepted: 3 January 2014

Published: 9 January 2014

\section{References}

1. Uchino S, Kellum JA, Bellomo R, Doig GS, Morimatsu H, Morgera S, Schetz M, Tan I, Bouman C, Macedo E, Gibney N, Tolwani A, Ronco C, Beginning and Ending Supportive Therapy for the Kidney (BEST Kidney) investigators: Acute renal failure in critically ill patients: a multinational, multicenter study. JAMA 2005, 294:813-818.

2. Hoste EA, Clermont G, Kersten A, Venkataraman R, Angus DC, De Bacquer D, Kellum JA: RIFLE criteria for acute kidney injury are associated with hospital mortality in critically ill patients: a cohort analysis. Crit Care 2006, 10:R73-R83.

3. Bagshaw SM, George C, Dinu I, Bellomo R: A multi-center evaluation of the RIFLE criteria for early acute kidney injury in critically ill patients. Nephrol Dial Transplant 2008, 23:1203-1210.

4. Coca SG, Yusuf B, Shlipak MG, Garg AX, Parikh CR: Long-term risk of mortality and other adverse outcomes after acute kidney injury: a systematic review and meta-analysis. Am J Kidney Dis 2009, 53:961-973.
5. Li Q, Fang JY, Wang WP, Liu JH, Wang KK: Cystatin C and serum creatinine in estimating acute kidney injury of shock patients. World J Emerg Med 2010, 1:185-189.

6. Herget-Rosenthal S, Marggraf G, Hüsing J, Göring F, Pietruck F, Janssen O, Philipp T, Kribben A: Early detection of acute renal failure by serum cystatin C. Kidney Int 2004, 66:1115-1122.

7. Che M, Xie B, Xue S, Dai H, Qian J, Ni Z, Axelsson J, Yan Y: Clinical usefulness of novel biomarkers for the detection of acute kidney injury following elective cardiac surgery. Nephron Clin Pract 2010, 115:c66-c72.

8. Chung MY, Jun DW, Sung SA: Diagnostic value of cystatin C for predicting acute kidney injury in patients with liver cirrhosis. Korean J Hepatol 2010, 16:301-307.

9. Haase M, Bellomo R, Devarajan P, Ma Q, Bennett MR, Möckel M, Matalanis G, Dragun $D$, Haase-Fielitz A: Novel biomarkers early predict the severity of acute kidney injury after cardiac surgery in adults. Ann Thorac Surg 2009, 88:124-130.

10. Ling Q, Xu X, Li JJ, Chen J, Shen JW, Zheng SS: Alternative definition of acute kidney injury following liver transplantation: based on serum creatinine and cystatin C levels. Transplant Proc 2007, 39:3257-3260.

11. Soto K, Coelho S, Rodrigues B, Martins H, Frade F, Lopes S, Cunha L, Papoila AL, Devarajan P: Cystatin C as a marker of acute kidney injury in the emergency department. Clin J Am Soc Nephrol 2010, 5:1745-1754.

12. Briguori C, Visconti G, Rivera NV, Focaccio A, Golia B, Giannone R, Castaldo D, De Micco F, Ricciardelli B, Colombo A: Cystatin C and contrast-induced acute kidney injury. Circulation 2010, 121:2117-2122.

13. Liang XL, Shi W, Liu SX, Yan LI, Xuan HJ, Xiong WP, Peng YQ, Huang JS, Liang YZ: Prospective study of cystatin C for diagnosis of acute kidney injury after cardiac surgery. Nan Fang Yi Ke Da Xue Xue Bao 2008, 28:2154-2156.

14. Nejat M, Pickering JW, Walker RJ, Endre ZH: Rapid detection of acute kidney injury by plasma cystatin $\mathrm{C}$ in the intensive care unit. Nephrol Dial Transplant 2010, 25:3283-3289.

15. Royakkers AA, Korevaar JC, van Suijlen JD, Hofstra LS, Kuiper MA, Spronk PE, Schultz MJ, Bouman CS: Serum and urine cystatin C are poor biomarkers for acute kidney injury and renal replacement therapy. Intensive Care Med 2011, 37:493-501.

16. Zhang Z, Lu B, Sheng X, Jin N: Cystatin C in prediction of acute kidney injury: a systemic review and meta-analysis. Am J Kidney Dis 2011, 58:356-365.

17. Abrahamson $M$, Olafsson I, Palsdottir $A$, Ulvsbäck $M$, Lundwall $A$, Jensson $O$, Grubb A: Structure and expression of the human cystatin C gene. Biochem J 1990, 268:287-294.

18. Schmid C, Ghirlanda-Keller C, Zwimpfer C, Zoidis E: Triiodothyronine stimulates cystatin C production in bone cells. Biochem Biophys Res Commun 2012, 419:425-430.

19. Kotajima N, Yanagawa Y, Aoki T, Tsunekawa K, Morimura T, Ogiwara T, Nara M, Murakami M: Influence of thyroid hormones and transforming growth factor- $\beta 1$ on cystatin C concentrations. J Int Med Res 2010, 38:1365-1373

20. Fricker $M$, Wiesli $P$, Brändle $M$, Schwegler B, Schmid C: Impact of thyroid dysfunction on serum cystatin C. Kidney Int 1944-1947, 2003:63.

21. Wiesli P, Schwegler B, Spinas GA, Schmid C: Serum cystatin C is sensitive to small changes in thyroid function. Clin Chim Acta 2003, 338:87-90.

22. Manetti L, Pardini E, Genovesi M, Campomori A, Grasso L, Morselli LL, Lupi I, Pellegrini $G$, Bartalena L, Bogazzi F, Martino E: Thyroid function differently affects serum cystatin $C$ and creatinine concentrations. J Endocrinol Invest 2005, 28:346-349.

23. Goede DL, Wiesli P, Brandle M, Bestmann L, Bernays RL, Zwimpfer C, Schmid C: Effects of thyroxine replacement on serum creatinine and cystatin $C$ in patients with primary and central hypothyroidism. Swiss Med Wkly 2009, 139:339-344.

24. Ozden TA, Tekerek H, Baş F, Darendeliler F: Effect of hypo-and euthyroid status on serum cystatin C levels. I Clin Res Pediatr Endocrinol 2010 2:155-158.

25. Bagshaw SM, Bellomo R: Cystatin C in acute kidney injury. Curr Opin Crit Care 2010, 16:533-539

26. Soni SS, Pophale R, Ronco C: New biomarkers for acute renal injury. Clin Chem Lab Med 2011, 49:1257-1263.

27. Mcllroy DR, Wagener $G$, Lee HT: Biomarkers of acute kidney injury: an evolving domain. Anesthesiology 2010, 112:998-1004. 
28. Pickering J, Endre ZH: Back-calculating baseline creatinine with MDRD misclassifies acute kidney injury in the intensive care unit. Clin J Am Soc Nephrol 2010, 5:1165-1173

29. Hanley JA, MCNeil BJ: A method of comparing the areas under ROC curves derived from same cases. Radiology 1983, 148:839-843.

30. Wang F, Pan W, Wang H, Wang S, Pan S, Ge J: Relationship between thyroid function and ICU mortality: a prospective observation study. Crit Care 2012, 16:R11.

31. Economidou F, Douka E, Tzanela M, Nanas S, Kotanidou A: Thyroid function during critical illness. Hormones (Athens) 2011, 10:117-124.

32. Knight EL, Verhave JC, Spiegelman D, Hillege HL, de Zeeuw D, Curhan GC, de Jong PE: Factors influencing serum cystatin $C$ levels other than renal function and the impact on renal function measurement. Kidney Int 2004, 65:1416-1421.

doi:10.1186/cc13186

Cite this article as: Wang et al:: The impacts of thyroid function on the diagnostic accuracy of Cystatin $\mathrm{C}$ to detect acute kidney injury in ICU patients: a prospective, observational study. Critical Care 2014 18:R9.

\section{Submit your next manuscript to BioMed Central and take full advantage of:}

- Convenient online submission

- Thorough peer review

- No space constraints or color figure charges

- Immediate publication on acceptance

- Inclusion in PubMed, CAS, Scopus and Google Scholar

- Research which is freely available for redistribution 